\title{
Bortezomib plus dexamethasone induction followed by autologous stem cell transplantation in multiple myeloma: A study from India
}

\author{
Jeevan Kumar', Sachin Minhas², Kamini Khillan³, Manorama Bhargava ${ }^{4}$, \\ Shyam Aggarwal ${ }^{5}$ \\ ${ }^{1}$ Senior Resident, Department of Hematology, Sir Ganga Ram Hospital, New Delhi, India, ${ }^{2}$ Scientist, Department of \\ Medical Oncology, Sir Ganga Ram Hospital, New Delhi, India, ${ }^{3}$ Consultant, Department of Transfusion Medicine, \\ Sir Ganga Ram Hospital, New Delhi, India, ${ }^{4}$ Senior consultant, Department of Hematology, Sir Ganga Ram Hospital, \\ India, ${ }^{5}$ Senior Consultant, Department of Medical Oncology, Sir Ganga Ram Hospital, New Delhi, India
}

A B S T R A C T

Background: The use of novel agents for induction prior to autologous stem cell transplantation (ASCT) has considerably improved the complete response (CR) rate in multiple myeloma (MM) patients. There are very few studies from the developing countries on the use of novel agents followed by ASCT. Aims and Objectives: The current study was aimed for retrospective evaluation of the efficacy and response rates of induction with bortezomib (Velcade) plus dexamethasone (VD regimen) followed by ASCT in Indian patients. Materials and Methods: Ten patients with newly diagnosed, symptomatic MM who had received four cycles of VD induction before stem cell collection were evaluated. High dose melphalan was given for conditioning followed by stem cell transfusion. Thalidomide or lenalidomide was used as post-transplantation maintenance treatment. Results: Post VD induction, the overall response rate (ORR) was $90 \%$ including $20 \% \mathrm{CR}, 40 \%$ very good partial response (VGPR), and $30 \%$ partial response (PR). Post ASCT, the ORR was $100 \%$, including $80 \%$ CR and $20 \%$ VGPR. The 5-year overall survival and progression free survival rates were $65.6 \%$ and $57.1 \%$, respectively. Conclusions: The VD induction regimen was effective and well tolerated in this retrospective analysis of Indian patients with newly diagnosed MM. It significantly improved the post-induction and post-transplant response rates without affecting stem cell collection.

Key words: Bortezomib, Multiple myeloma, Stem cell transplantation, Novel agents

\section{Access this article online}

Website:

http://nepjol.info/index.php/AJMS

DOI: 10.3126/ajms.v7i4.14434

E-ISSN: 2091-0576

P-ISSN: 2467-9100

\section{INTRODUCTION}

Multiple myeloma (MM) is a malignant disorder characterized by the proliferation of a single clone of plasma cells derived from $\mathrm{B}$ cells in the bone marrow and is associated with an increased level of monoclonal proteins in blood and/urine. The management of multiple myeloma has improved greatly in past decade. Despite this, it continues to be an incurable disease. Since MM is an incurable disease, the main aim of the newer therapeutic options is improving the overall survival (OS) $\cdot{ }^{1-3}$ High dose therapy followed by autologous stem cell transplantation (HDT-ASCT) has been found to be very effective in the treatment of MM and is considered as gold standard for treating younger patients (patients up to 65 years of age). Randomized studies have shown considerable improvement in median survival with HDT-ASCT as compared to conventional chemotherapy. Patients achieving complete response (CR) or very good partial response (VGPR) appear to benefit more than those who had only a partial response. The main objective is therefore to achieve CR or VGPR which consequently will lead to improved progression free survival (PFS) and OS. ${ }^{4.8}$ 
In the past, standard induction therapy consisted of vincristine, doxorubicin, and dexamethasone (VAD) which resulted in $\mathrm{CR}$ rates $<10 \%$. However the introduction of novel agents like bortezomib, thalidomide and lenalidomide for induction has immensely helped in improving the outcomes. $^{2,3,9}$ In 2006, Harousseau et al in the Intergroupe Francophone du Myelome (IFM) study has examined bortezomib in combination with dexamethasone as induction prior to HDT-ASCT. The overall response rate (ORR) was 67\%, including a 31\% CR+VGPR rate, prior to transplant. Post-transplant, the ORR and CR+VGPR rates increased to $90 \%$ and $54 \%$, respectively. ${ }^{9}$

In 2010, Palumbo et al evaluated 102 patients for the effect of bortezomib as induction therapy before autologous transplantation, followed by lenalidomide as consolidation-maintenance in myeloma patients. In pretransplant analysis, after PAD (bortizomib, doxorubicin, dexamethasone), 58\% of patients had VGPR or better, including $13 \%$ with CR. Post-transplant, $82 \%$ of patients had at least VGPR, including 38\% with CR. After maintenance with lenalidomide, $86 \%$ of patients had at least VGPR and 66\% had CR. They concluded that bortezomib as induction before autologous transplantation, followed by lenalidomide as consolidation-maintenance, is an effective regimen. ${ }^{10}$

All this data is from the western countries. There are not many studies to establish the efficacy and safety of bortezomib in Indian population. In the current retrospective analysis, we sought to evaluate the efficacy and safety of bortezomib (Velcade) plus dexamethasone (VD regimen) in Indian patients with newly diagnosed MM.

\section{MATERIALS AND METHODS}

The medical records of ten patients with untreated, symptomatic MM who had received VD induction before stem cell collection were reviewed retrospectively. Informed consent was obtained from all patients. This study has been approved by the ethics committee of Sir Ganga Ram Hospital, New Delhi, India and has therefore been performed in accordance with the ethical standards laid down in the 1964 Declaration of Helsinki and its later amendments. The diagnosis of MM was confirmed using the International Myeloma Working Group (IMWG) criteria. $^{11}$

VD induction comprised of bortezomib $\left(1.3 \mathrm{mg} / \mathrm{m}^{2}\right)$ and dexamethasone $(40 \mathrm{mg})$ administered weekly. Each cycle consists of four such weekly bortezomib injections plus tablet dexamethasone. Each patient had received a total of four such cycles and monitored for response. Before each bortezomib dose, the patient was evaluated for possible toxicity according to the National Cancer Institute (NCI) Common Toxicity Criteria, version 2.0. If grade 4 hematological toxicity, febrile neutropenia, or any grade $\geq 3$ non-hematologic toxicity related to bortezomib occurred, bortezomib was withheld until toxicity returned to grade $\leq 1$ (excluding peripheral neuropathy). If the toxicity did not resolve within 2 weeks, bortezomib was discontinued. If the toxicity resolved, bortezomib was restarted at a dose reduced by $25 \%$. Bortezomib was to be discontinued in the event of grade 4 peripheral neuropathy.

Peripheral blood stem cell collection technique involved administration of granulocyte colony stimulating factor (G-CSF), $300 \mu \mathrm{g}$ twice daily for 5 days. At least $2.0 \times 10^{6}$ $\mathrm{CD} 34+$ cells $/ \mathrm{kg}$ were collected. Adequate number of stem cells was collected in nine patients by a single harvest. One patient required apharesis twice for adequate stem cell collection. These cells were cryo-preserved. High dose melphalan $\left(200 \mathrm{mg} / \mathrm{m}^{2}\right)$ was given as preparative regimen followed by stem cell transfusion. All patients were put on maintenance therapy, 6 patients received thalidomide $(50 \mathrm{mg} /$ day) and 4 patients received lenalidomide (10 mg/day).

\section{Response criteria}

Serum and urine protein electrophoresis were collected at baseline, after second and fourth cycle of bortezomib plus dexamethasone. Bone marrow examination and Immunofixation were done at baseline and at the time of documenting CR and VGPR. Response was assessed according to the International Myeloma Working Group (IMWG) uniform response criteria. ${ }^{11} \mathrm{~A}$ complete response (CR) was defined by negative immunofixation on the serum and urine, and the disappearance of any soft tissue plasmacytomas and $\leq 5 \%$ plasma cells in bone marrow. A very good partial response (VGPR) was defined as serum and urine M-protein detectable by immunofixation but not on electrophoresis, or $90 \%$ or greater reduction in serum M-protein plus urine M-protein level <100 mg per 24 h. A partial response (PR) was defined by a reduction of $\mathrm{M}$ protein in serum of at least $50 \%$ and a reduction in urine of at least $90 \%$ or urine $\mathrm{M}$ protein level $<200 \mathrm{mg}$ per $24 \mathrm{~h}$. Progressive disease (PD) was defined by any of the following: an increase of $\mathrm{M}$ protein in serum of more than $25 \%$ from baseline (the absolute increase must be $\geq 0.5 \mathrm{~g} / \mathrm{dl}$ ) or urine (the absolute increase must be $\geq 200 \mathrm{mg} / 24 \mathrm{~h}$ ), an increase in bone marrow plasma cells (the absolute $\%$ must be $\geq 10 \%$ ), new or increased bone lesions or plasmacytomas, or new hypercalcemia. ${ }^{12}$

\section{RESULTS}

Table 1 shows the characteristics of the patients before bortezomib therapy. The mean age of the patients was 51.7 years (Range: 42-72); eight were males while two were 
females. Six patients had IgG disease, one had IgA and three had light chain disease. The median CD34-positive stem cell count was $4.85 \times 10^{6} / \mathrm{kg}$. All the patients engrafted post transplant. The median time for engraftment (absolute neutrophil count $>500 / \mu \mathrm{L}$ for 3 consecutive days) was 10.5 days and median time for platelet count $>20000 / \mu \mathrm{L}$ was 11.5 days.

Table 2 shows the response rates to treatment. After induction with VD protocol, the overall response rate (ORR) was $90 \%$. Two patients $(20 \%)$ had a CR, four patients (40\%) had VGPR, three patients $(30 \%)$ had PR and one patient $(10 \%)$ had PD. Post ASCT, the patient with PD achieved VGPR. All patients with PR and three out of four patients with VGPR achieved CR making the total responses as eight CRs and two VGPRs. Thus, overall response rate was $100 \%$ post ASCT, including $80 \%$ CR and $20 \%$ VGPR. All three patients with renal insufficiency experienced improvement in renal function and did not require dialysis post-ASCT.

Three patients $(30 \%)$ had a relapse post-ASCT; two of them (one patient had VGPR and another had CR) relapsed after 14 months, and the third patient (in VGPR) relapsed after 8 months. Three patients (30\%) expired, two relapsed patients (one expired after 16 months and another after 30 months of transplant) died due to progressive myeloma and the third patient (in CR expired after 12 months of transplant) due to an unrelated cause (Dengue hemorrhagic

\begin{tabular}{lc} 
Table 1: Patient characteristics $(\boldsymbol{n = 1 0})$ & \\
\hline Patients, $n$ & 10 \\
Mean age in years, (range) & $51.7(42-72)$ \\
Male/female $(\%)$ & $8 / 2(80 / 20)$ \\
Mean weight $(\mathrm{kg})$ & 71.3 \\
Renal function at diagnosis, $n(\%)$ & \\
Normal & $7(70 \%)$ \\
Abnormal (serum creatinine $\geq 2 \mathrm{mg} / \mathrm{dl})$ & $3(30 \%)$ \\
Myeloma subtype, $n(\%)$ & \\
IgA-kappa & $1(10.0 \%)$ \\
IgG-kappa & $3(30.0 \%)$ \\
IgG-lamda & $3(30.0 \%)$ \\
Light chain & $3(30.0 \%)$ \\
Median CD34+ cell count & $4.85 \times 10 \% / \mathrm{kg}$ \\
\hline
\end{tabular}

\begin{tabular}{|c|c|c|c|c|c|}
\hline \multirow[t]{2}{*}{ Response } & \multirow{2}{*}{$\begin{array}{c}\text { Post VD } \\
\text { induction, } \\
n(\%)\end{array}$} & \multicolumn{2}{|c|}{ Post ASCT, $n(\%)$} & \multirow{2}{*}{ Relapse } & \multirow[t]{2}{*}{ Expirec } \\
\hline & & CR & VGPR & & \\
\hline CR & $2(20 \%)$ & 2 & - & - & - \\
\hline VGPR & $4(40 \%)$ & 3 & 1 & 1 & 1 \\
\hline PR & $3(30 \%)$ & 3 & - & 1 & 2 \\
\hline PD & $1(10 \%)$ & - & 1 & 1 & - \\
\hline Total (\%) & 10 & \multicolumn{2}{|c|}{$8(80 \%) 2(20 \%)$} & $3(30 \%)$ & $3(30 \%)$ \\
\hline $\begin{array}{l}\text { ORR } \\
(C R+V G P R+P R)\end{array}$ & $9(90 \%)$ & \multicolumn{2}{|c|}{$10(100 \%)$} & - & - \\
\hline
\end{tabular}

fever). The overall survival rate at 6 months was $100 \%$. One year OS rate was $90 \%$ and two and five year OS rates were $78.8 \%$ and $65.6 \%$ respectively. The PFS rate at 6 months was $100 \%$, at one year was $80 \%$ and 5 year PFS rate was $57.1 \%$ (Figures 1 and 2).

Two patients $(20 \%)$ developed grade 2 neuropathy and two patients $(20 \%)$ developed herpes zoster infection due to bortezomib therapy. Three patients developed thrombocytopenia $(30 \%)$ requiring dose modification of bortezomib. All the patients developed fever post-ASCT; one patient was diagnosed with malaria (Plasmodium vivax), one patient had blood culture positive for Staphylococcus aureus and another patient had blood culture positive for Klebsiella pneumoniae. All were successfully managed for fever and infections with antibiotics, antifungals, antimalarials and supportive treatment.

\section{DISCUSSION}

The main objective of induction therapy in patients eligible for ASCT is reducing the tumor mass prior to the high

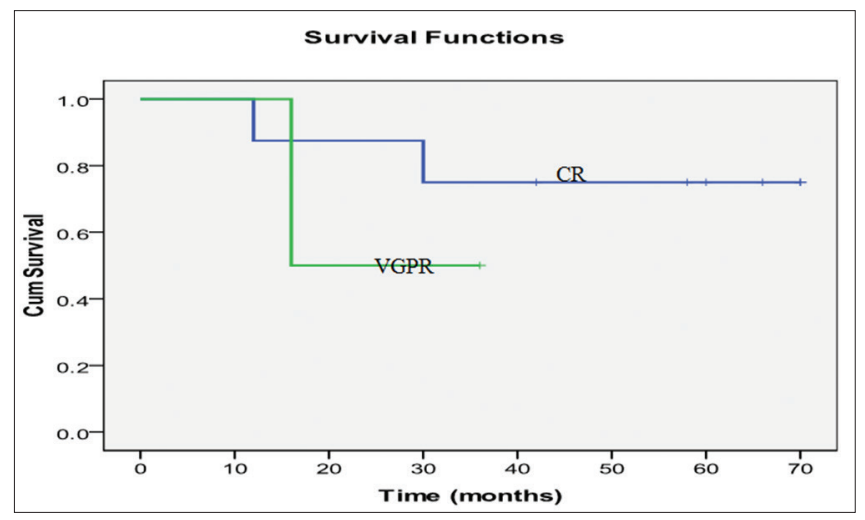

Figure 1: Overall survival in patients of complete response (CR) and very good partial response (VGPR)

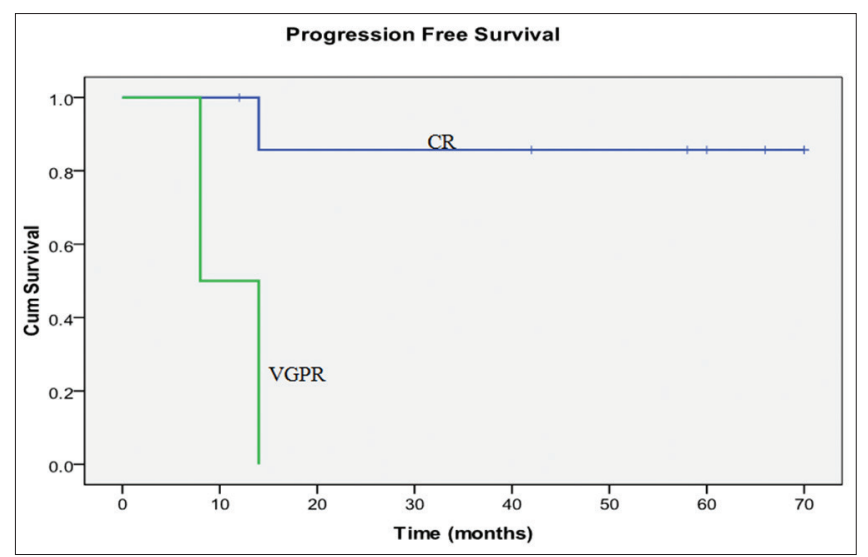

Figure 2: Progression free survival in patients of complete response (CR) and very good partial response (VGPR) 
dose therapy and achieving highest possible response rate without any adverse impact on the stem cell mobilization or the hematopoietic graft. In this context, VD regimen has been found to be effective and has become an important part of the standard induction therapy., ${ }^{9,13,14}$ The data on the use of HDT-ASCT in Indian population is very scarce and that on bortezomib is even lesser. There is limited information regarding the efficacy, adverse effects or the long term complications of the induction regimens. ${ }^{15}$ Therefore we sought to evaluate the efficacy and safety of the bortezomib plus dexamethasone as induction treatment followed by ASCT in newly diagnosed patients with MM.

Although patients under 65 yrs of age are usually considered to be suitable for ASCT, this arbitrary cutoff does not completely exclude older patients. Selected patients up to 75 yrs of age who are medically fit can be considered for ASCT. ${ }^{16}$ Accordingly, in this retrospective analysis of patients with newly diagnosed MM, one $72 \mathrm{yr}$ old patient was successfully administered HDT-ASCT. The patient achieved CR without any major adverse events.

High response rates (overall response rate was 90\% including CR in 20\% patients) were observed in this study with the VD induction regimen. These response rates were similar to observed in previous studies with the VD regimen ${ }^{9,10}$ but are higher than those obtained in studies with conventional chemotherapy. ${ }^{4-8,13}$ Superiority of novel agents-based induction therapy both in terms of mobilization of stem cells and response rates to ASCT has been established. ${ }^{1}$ Other studies showed that better pre-transplantation remission status implies better posttransplant CR rate. ${ }^{6}$ Therefore, the choice of pre-transplant induction therapy is particularly important.

In this study post transplant overall response rate was $100 \%$, including $80 \% \mathrm{CR}$ and $20 \%$ VGPR. These response rates were higher than that observed in other similar studies. ${ }^{910}$ These variations may be due to less number of total patients in our study. Achievement of CR in myeloma represents the major surrogate marker for long-term OS and PFS. ${ }^{67}$ Harousseau et al identified achievement of 'at least VGPR' as an important predictor of outcome in an analysis of 802 patients. ${ }^{17}$ In the present study the 5 -year overall survival and progression free survival rates were $65.6 \%$ and $57.1 \%$, respectively.

No irreversible toxicities were seen. The adverse events reported were comparable to other studies. All the adverse events were manageable using dose adjustments and supportive therapy. Thrombocytopenia recovered following dose modification of bortezomib. The most common non-hematological adverse events were infections. Prompt assessment of the fever was done and antibiotics were instituted appropriately. Use of dexamethasone in induction therapy may cause infection due to suppression of cell mediated immunity. In our study, dexamethasone was used every weekly with bortezomib injection, instead of the usual high dose (3 cycles of 4 days each, every month). Lower cumulative doses of dexamethasone have been found to significantly decrease grade 3-4 infections. ${ }^{18}$ Two patients $(20 \%)$ had peripheral neuropathy. Peripheral neuropathy is a frequently seen adverse effect with the use of bortezomib. In various trials around 31 to $37 \%$ of the patients suffered from peripheral neuropathy. ${ }^{19-21}$

Maintenance treatment was given to all patients, with either thalidomide or lenalidomide. After achievement of $\mathrm{CR}$, its maintenance is equally important in the treatment of MM. Loss of CR early after transplant has been found to be a poor prognostic indicator for PFS and OS. Both thalidomide and lenalidomide have been shown to be effective agents for maintenance therapy. ${ }^{22-24}$

\section{CONCLUSION}

VD regimen prior to ASCT obtained good response rates, without impairment of stem cell collection. Response to induction therapy is related to better result after ASCT and, finally, into longer survival. From this retrospective analysis, it can be stated that Indian patients with untreated, symptomatic MM can be effectively and safely treated with VD induction followed by ASCT. However, only ten patients were evaluated in this study and in order to confirm the results of this study, larger studies are required.

\section{ACKNOWLEDGMENTS}

The authors thank the patients and families who participated in this study, the physicians and nurses for their input in performing this study.

\section{REFERENCES}

1. Kyle RA and Rajkumar SV. Multiple myeloma. Blood 2008; 111(6):2962-2972.

2. Bladé J, Cibeira MT, Fernández de Larrea $C$ and Rosiñol L. Multiple myeloma. Ann Oncol 2010; 21 Suppl 7:vii 313-319.

3. Rajkumar SV. Multiple Myeloma: 2011 update on diagnosis, risk-stratification, and management. Am J Hematol 2011; 86(1):57-65.

4. Barlogie B, Shaughnessy J, Tricot G, Jacobson J, Zangari M, Anaissie E, et al. Treatment of multiple myeloma. Blood 2004; 103(1):20-32.

5. Gupta A and Kumar L. Evolving role of high dose stem cell therapy in multiple myeloma. Indian J Med Paediatr Oncol 2011; 32(1):17-24.

6. Harousseau JL, Attal $\mathrm{M}$ and Avet-Loiseau $\mathrm{H}$. The role of complete response in multiple myeloma. Blood 2009; 114(15):3139-3146. 
7. Van de Velde H, Liu X, Chen G, Cakana A, Deraedt W and Bayssas M. Complete response correlates with long-term survival and progression-free survival in high-dose therapy in multiple myeloma. Haematologica 2007; 92: 1399-1406.

8. Kyle RA, Leong T, Li S, Oken MM, Kay NE, Van Ness B, et al. Complete response in multiple myeloma: clinical trial E9486, an Eastern Cooperative Oncology Group study not involving stem cell transplantation. Cancer 2006; 106(9):1958-1966.

9. Harousseau JL, Attal M, Leleu X, Troncy J, Pegourie B, Stoppa AM, et al. Bortezomib plus dexamethasone as induction treatment prior to autologous stem cell transplantation in patients with newly diagnosed multiple myeloma: results of an IFM phase II study. Haematologica 2006; 91(11):1498-1505.

10. Palumbo A, Gay F, Falco P, Crippa C, Montefusco V, Patriarca F, et al. Bortezomib as induction before autologous transplantation, followed by lenalidomide as consolidation-maintenance in untreated multiple myeloma patients. J Clin Oncol 2010; 28:800-807.

11. Kyle RA, Child JA, Anderson K, Barlogie B, Bataille $R$, Bensinger W, et al. Criteria for the classification of monoclonal gammopathies, multiple myeloma and related disorders: a report of the International Myeloma Working Group. $\mathrm{Br} \mathrm{J}$ Haematol 2003; 121(5):749-757.

12. Durie BG, Harousseau JL, Miguel JS, Bladé J, Barlogie B, Anderson $\mathrm{K}$, et al. International Myeloma Working Group. International uniform response criteria for multiple myeloma. Leukemia 2006; 20(9):1467-1473.

13. Harousseau JL, Attal M, Avet-Loiseau H, Marit G, Caillot D, Mohty $\mathrm{M}$, et al. Bortezomib plus dexamethasone is superior to vincristine plus doxorubicin plus dexamethasone as induction treatment prior to autologous stem-cell transplantation in newly diagnosed multiple myeloma: results of the IFM 2005-01 phase III trial. J Clin Oncol 2010; 28(30):4621-4629.

14. Kortüm $M$ and Einsele $H$. Results of the first bortezomib-based induction therapy in the treatment of multiple myeloma. Expert Opin Pharmacother 2011; 12(10):1661-1663.

15. Kumar L, Ghosh J, Ganessan P, Gupta A, Hariprasad R and Kochupillai V. High-dose chemotherapy with autologous stem cell transplantation for multiple myeloma: what predicts the outcome? Experience from a developing country. Bone Marrow Transplant 2009; 43(6):481-489.

16. Cavo M, Rajkumar SV, Palumbo A, Moreau P, Orlowski R,
Bladé J, et al. International Myeloma Working Group. International Myeloma Working Group consensus approach to the treatment of multiple myeloma patients who are candidates for autologous stem cell transplantation. Blood 2011; 117(23):6063-6073.

17. Harousseau JL, Avet-Loiseau $\mathrm{H}$, Attal M, Charbonnel C, Garban F, Hulin C, et al. Achievement of at least very good partial response is a simple and robust prognostic factor in patients with multiple myeloma treated with high-dose therapy: long-term analysis of the IFM 99-02 and 99-04 trials. J Clin Oncol 2009; 27:5720-5726.

18. Rajkumar SV, Jacobus S, Callander NS, Fonseca R, Vesole DH, Williamns ME, etal. Lenalidomide plus high-dose dexamethasone versus lenalidomide plus low-dose dexamethasone as initial therapy for newly diagnosed multiple myeloma: an open-label randomised controlled trial. Lancet Oncol 2010; 11(1):29-37.

19. Richardson PG, Barlogie B, Berenson J, Singhal S, Jagannath S, Irwin $\mathrm{D}$, et al. A phase 2 study of bortezomib in relapsed, refractory myeloma. N Engl J Med 2003; 348(26):2609-2617.

20. Jagannath S, Barlogie B, Berenson J, Siegel D, Irwin D, Richardson PG, et al. A phase 2 study of two doses of bortezomib in relapsed or refractory myeloma. $\mathrm{Br} \mathrm{J}$ Haematol 2004;127:165-172.

21. Richardson PG, Sonneveld $P$, Schuster MW, Irwin D, Stadtmauer EA, Facon $T$, et al. Assessment of Proteasome Inhibition for Extending Remissions (APEX) Investigators. Bortezomib or high-dose dexamethasone for relapsed multiple myeloma. N Engl J Med 2005; 352:2487-2498.

22. Attal M, Harousseau JL, Leyvraz S, Doyen C, Hulin C, Benboubker L, et al. Inter-Groupe Francophone du Myélome (IFM). Maintenance therapy with thalidomide improves survival in patients with multiple myeloma. Blood 2006; 108(10):3289-3294.

23. McCarthy PL, Owzar K, Anderson KC, Hofmeister CC, Hassoun H, Hurd DD, et al. Phase III intergroup study of lenalidomide versus placebo maintenance therapy following single autologous stem cell transplant (ASCT) for multiple myeloma (MM): CALGB 100104. J Clin Oncol 2010; 28:15s, (suppl; abstr 8017).

24. Attal M, Lauwers VC, Marit G, Denis Caillot, Facon T, Hulin $C$, et al. Maintenance treatment with lenalidomide after transplantation for MYELOMA: Final analysis of the IFM 200502. ASH Annual Meeting Abstracts. Blood 2010; 116(21):310.

\section{Authors Contribution:}

JK-Concept and design of the study, clinical data collection and interpretation, statistical analysis, reviewed the literature, manuscript preparation and critical revision of the manuscript; SM-Study design, data collection and interpretation, reviewed the literature, manuscript revision; KK-Literature search, data collection and interpretation, manuscript revision; MB-Literature search, data collection and interpretation, manuscript revision; SA-Concept and design of the study, data collection and interpretation, reviewed the literature, manuscript revision.

Source of Support: Nil, Conflict of Interest: None declared. 